\title{
Multiscale modeling of coastal, shelf, and global ocean dynamics
}

\author{
Pierre F. J. Lermusiaux • Jens Schröter • Sergey Danilov • \\ Mohamed Iskandarani • Nadia Pinardi • Joannes J. Westerink
}

Received: 9 September 2013 / Accepted: 12 September 2013 / Published online: 29 September 2013

(C) Springer-Verlag Berlin Heidelberg 2013

In contemporary ocean science, modeling systems that integrate understanding of complex multiscale phenomena and utilize efficient numerics are paramount. Many of today's fundamental ocean science questions involve multiple scales and multiple dynamics. A new generation of modeling systems would allow to study such questions quantitatively by being less restrictive dynamically and more efficient numerically than more traditional systems.

Such multiscale ocean modeling is the theme of this topical collection. Two large international workshops were organized on this theme, one in Cambridge, USA (IMUM2010), and one in Bremerhaven, Germany (IMUM2011). Contributions from

Responsible Editor: Jörg-Olaf Wolff

P. F. J. Lermusiaux $(\bowtie)$

Department of Mechanical Engineering, Massachusetts Institute of Technology, Room 5-207B, 77 Massachusetts Avenue, Cambridge, MA 02139-4307, USA

e-mail: pierrel@mit.edu

J. Schröter · S. Danilov

Alfred Wegener Institute for Polar and Marine Research,

Bussestrasse 24, D-27570 Bremerhaven, Germany

M. Iskandarani

MPO Division, Rosenstiel School for Marine and Atmospheric

Sciences, University of Miami, Miami, FL 33149, USA

\section{N. Pinardi}

University of Bologna Laboratorio SINCEM, Laboratori R. Sartori

Via S. Alberto 163, 48123 Ravenna, Italy

\section{N. Pinardi}

Istituto Nazionale di Geofisica e Vulcanologia, Via Aldo Moro 44, 40127 Bologna, Italy

\section{J. J. Westerink}

Department of Civil and Environmental Engineering and Earth Sciences, University of Notre Dame, 156a Fitzpatrick Hall, Notre Dame, IN 46556-0767, USA the scientific community were encouraged on all aspects of multiscale ocean modeling from ocean science and dynamics to the development of new computational methods and systems. Building on previous meetings (e.g., Deleersnijder and Lermusiaux 2008; Deleersnijder et al. 2010), the workshop discussions and the final contributions to the topical collection are summarized next.

The scientific application domains discussed and presented ranged from estuaries to the global ocean, including coastal regions and shelf seas. Multi-resolution modeling of physical, biological, chemical, and sea ice processes as well as air-sea interactions were described. The multiscale dynamics considered involved hydrostatic, non-hydrostatic, turbulent, and sea surface processes.

Computational results and discussions emphasized multiresolution simulations using unstructured and structured meshes, aiming to widen the range of resolved scales in space and time. They included finite volume and finite element spatial discretizations, high-order schemes, preconditioners, solver issues, grid generation, adaptive modeling, data assimilation, coupling with atmospheric or biogeochemical models, and distributed computing. The advantages of using unstructured meshes and related approaches in particular multi-grid embedding, nesting systems, wavelets, and other multiscale decompositions were discussed. Techniques for the study of multi-resolution results, visualization, optimization, model evaluations, and uncertainty quantification were also examined.

\section{Multiscale interdisciplinary dynamics}

A number of manuscripts in the topical collection focus on multiscale interdisciplinary dynamics, specifically ocean physics and ice interactions, physical-biogeochemical interactions, and physical-sediment-coast interactions. 
Two articles address the study of ocean circulations and ice interactions utilizing unstructured finite element models. Specifically, Wang et al. (2012) set up and evaluate the performance of the Finite Element Sea-ice Ocean Model (FESOM) in the North Atlantic and Arctic Ocean region in a hindcast experiment. The authors argue that their simulations indicate that variability of the Atlantic meridional overturning circulation (AMOC) can be explained by variability of the thermohaline forcing over deep convection sites. The authors complete a water-hosing experiment to study the model sensitivity to increased freshwater input from Greenland Ice Sheet melting. This simulated experiment leads to changes in the ocean circulation and local dynamical sea level. The authors also compare their results to these of previous modeling studies. In a companion global study, Scholz et al. (2013) utilize FESOM to study inter-annual to decadal variability in deep-water formation rates over the global ocean for the period 1958-2004. Higher resolution is utilized in the known deep-water formation areas. Comparing their multi-resolution simulation to varied ocean data, the authors find that the simulated sea-ice distributions and transport as well as the hydrographic structures are in relatively good agreement with observations. They find significant variability in the AMOC as well as in the properties of the North Atlantic deep-water formation areas.

Considering next physical-biogeochemical interactions, Khangaonkar et al. (2012) combine the Finite Volume Coastal Ocean Model with the Integrated Compartment Model (CE-QUAL-ICM) water quality kinetics to characterize annual biogeochemical cycles and nutrient balances in Puget Sound. Using this combined system, the authors simulate phytoplankton biomass growth and characterize the relative contributions from natural and anthropogenic sources and from exchanges with the Pacific Ocean. Results based on 2006 data indicate that biogeochemical cycle properties are strongly correlated with seasonal variations of physical properties. Specifically, the authors discuss the effects of seasonal and depth-dependent variability in the exchanges with the Pacific Ocean. This work shows that unstructured grid models can be applied to ecosystem assessment studies.

Considering ocean flows and seashore interactions, Vousdoukas et al. (2012) calibrate a dune-erosion model, $\mathrm{XBeach}$, to predict beach-profile morphodynamic responses to storm events of the coast of Portugal and discuss potential operational applications. Offshore wave and tidal data are inputs to a SWAN wave model, which provides wave conditions to XBeach. Simulations using this nested SWAN/XBeach system are compared to data and, after XBeach calibration, the performance is satisfactory. Results highlight the fragility of the local dune field in response to storms. To illustrate the potential for an early warning system, the authors force the SWAN/
XBeach models with an existing operational forecast WAVEWATCH-III/SWAN model. Considering finerscales, Plomaritis and Collins (2013) study the smallscale modifications of tidally driven flows caused by hydro- and sediment dynamics in the near-field area of offshore breakwaters. The offshore breakwaters are located on the West Sussex coastline, south coast of England. The computations concentrate on modifications caused by the breakwater structures in the absence of waves using a shallow water hydrodynamic modeling system. Modifications of tidal currents and bedload transports are linked to the obstruction of the main tidal flow and to flow channelization between the structures and the coastline. The authors argue that to predict the morphological evolution of coastlines protected by structures, both tidal and wave processes have to be modeled.

Considering coastal ocean responses to storm events, Otero et al. (2013) analyze the consequences of a stormy winter period (2009/2010) on the dynamics off Northwest Iberia, utilizing the Regional Oceanic Modeling System with mesh refinement and a nesting procedure (AGRIF). The multi-resolution results show how the frequent weather storms forced river plumes in fast jet structures, followed by offshore expansion of the plumes due to wind reversal behind the storm. The authors compare simulations and data and utilize Lagrangian modeling experiments to predict a northward displacement of surface particles after several wind events.

\section{Multi-resolution meshes and unstructured modeling}

A prerequisite to multi-resolution modeling is the creation of high-quality unstructured meshes or nested grids that allow the accurate representation of geometry-related dynamics. Several manuscripts focus on such research.

Conroy et al. (2012) develop and illustrate an advanced, automatic, unstructured mesh generator for shallow water models. The two-dimensional mesh generator automatically produces high-quality meshes from a minimal set of inputs, specifically the points defining the geometry and the target minimum and maximum element sizes. Inspired by Persson and Strang (2004), local element sizes are optimized for the local bathymetry, coastline, and expected solutions, ensuring consistency through multiple iterations. Nodes are thought to be connected with springs, and the system is integrated to springbased force quasi-equilibrium. Techniques used include a signed distance function, the approximation of coastlines by smooth cubic splines, and a mesh function to control element sizes. In a complementary investigation, Terwisscha van Scheltinga et al. (2012) present an efficient mesh generation scheme designed for highly 
irregular oceanic domains such as archipelagos. The new approach is based on a standard mesh size field that depends only on the desired resolution and proximity to the nearest coastline. Examples are provided for the Aegean Sea, Indonesian Archipelago and Canadian Arctic Archipelago.

Reckinger et al. (2012) develop several adaptive volume penalization techniques to represent topographic effects in ocean models that use a stair-step representation of the geometry. Such stair-steps can cause accuracy and numerical stability problems. The authors utilize Brinkman penalization, adding a term to the governing equations, to enforce no slip boundary conditions. All governing equations are then solved on temporally and spatially varying meshes using the adaptive wavelet collocation method. The authors analyze their joint use of Brinkman penalization and adaptive wavelet collocation for the shallow water equations and present preliminary results for primitive equations.

Viré et al. (2012) model fluid-structure interactions by coupling a finite element fluid/ocean model (Fluidity-ICOM) with a combined finite-discrete element solid model (Y3D). Different unstructured meshes are used for the fluids and solids. A function super-space of these meshes is employed to ensure conservative forces at the discrete level. Spatial conservation is shown to be independent of the mesh resolutions while accuracy is shown to require fine resolutions in both meshes. The approach is evaluated on several examples. The authors argue that it enables accurate simulations of fluid loading and vortex-induced vibrations in ocean engineering structures.

Finally, efficient finite element schemes for accurate fourdimensional ocean simulations are presented. Aizinger et al. (2013) apply a 3D-in-space discontinuous Galerkin finite element model to simulate baroclinic circulations in Corpus Christi Bay, Texas and the surrounding regions. The authors describe their modeling system, the University of Texas Bays and Estuaries 3D (UTBEST3D) simulator, which is based on a discontinuous Galerkin scheme for unstructured prismatic meshes. A new feature is the extension to baroclinic flows. The authors show that simulations compare well with data sets collected for the year 2000. They also describe their new turbulence scheme and their localized vertical discretization. Simulations are conducted on parallel clusters, and an evaluation of parallel performance is provided.

\section{Conclusions}

This topical collection shows that much progress has been made in the utilization of unstructured numerical techniques for the multiscale modeling of physical, biological, seabed, and sea-ice interactions. High-quality multi-resolution meshes and grids are needed for accurate modeling and methods for mesh generation have been developed and applied. The significance of accurate multi-resolution modeling for four-dimensional multiscale ocean studies was stressed. Directions for future progress include the utilization of higher order schemes in time and space, as well as the efficient adaptive discretization and distributed implementation of realistic unstructured modeling systems. Today, key ocean science questions involve multiple scales and nonlinear dynamics and such systems are most likely to answer them.

Acknowledgments The guest editors are indebted to the authors and anonymous reviewers for their contributions to this topical collection. Pierre Lermusiaux is very grateful to the Office of Naval Research for support under grants N00014-08-1-1097 and N00014-12-1-0944 to MIT and the National Science Foundation for support under grant OCE1061160 to MIT. Some of the articles of this topical collection derive from presentations made at the 9th and 10th International Workshops on Multiscale (Un)-structured mesh numerical Modeling for coastal, shelf and global ocean dynamics (IMUM2010, 17-20 August 2010 at MIT, Cambridge, USA and IMUM2011, 22-25 August 2011 at Bremerhaven, Germany).

\section{References}

Aizinger V, Proft J, Dawson C, Pothina D, Negusse S (2013) A threedimensional discontinuous Galerkin model applied to the baroclinic simulation of Corpus Christi Bay. Ocean Dyn 63:89-113. doi:10.1007/ s10236-012-0579-8

Conroy CJ, Kubatko EJ, West DW (2012) ADMESH: an advanced, automatic unstructured mesh generator for shallow water models. Ocean Dyn 62:1503-1517. doi:10.1007/s10236-012-0574-0

Deleersnijder E, Lermusiaux PFJ (2008) Multi-scale modelling: nested grid and unstructured mesh approaches. Ocean Dyn 58:335-336. doi:10.1007/s10236-008-0170-5

Deleersnijder E, Legat V, Lermusiaux PFJ (2010) Multi-scale modelling of coastal, shelf and global ocean dynamics. Ocean Dyn 60:13571359. doi:10.1007/s10236-010-0363-6

Khangaonkar T, Sackmann B, Long W, Mohamedali T, Roberts M (2012) Simulation of annual biogeochemical cycles of nutrient balance, phytoplankton bloom(s), and DO in Puget Sound using an unstructured grid model. Ocean Dyn 62:1353-1379. doi:10.1007/s10236012-0562-4

Otero P, Ruiz-Villarreal M, García-García L, González-Nuevo G, Cabanas J-M (2013) Coastal dynamics off Northwest Iberia during a stormy winter period. Ocean Dyn 63:115-129. doi:10.1007/ s10236-012-0585-x

Persson PO, Strang G (2004) A simple mesh generator in MATLAB. SIAM Rev 46:329-345

Plomaritis T, Collins MB (2013) Hydrodynamic and sediment dynamic modifications of tidal flow in the near-field area of offshore breakwaters. Ocean Dyn 63:225-241. doi:10.1007/ s10236-013-0592-6

Reckinger M, Vasilyev OV, Fox-Kemper B (2012) Adaptive volume penalization for ocean modeling. Ocean Dyn 62:1201-1215. doi: 10.1007/s10236-012-0555-3

Scholz P, Lohmann G, Wang Q, Danilov S (2013) Evaluation of a FiniteElement Sea-Ice Ocean Model (FESOM) set-up to study the interannual to decadal variability in the deep-water formation rates. Ocean Dyn 63:347-370. doi:10.1007/s10236-012-0590-0 
Terwisscha van Scheltinga AD, Myers PG, Pietrzak JD (2012) Mesh generation in archipelagos. Ocean Dyn 62:1217-1228. doi:10.1007/ s10236-012-0559-Z

Viré A, Xiang J, Milthaler F, Farrell PE, Piggott MD, Latham J-P, Pavlidis D, Pain CC (2012) Modelling of fluid-solid interactions using an adaptive-mesh fluid model coupled with a combined finitediscrete element model. Ocean Dyn 62:1487-1501. doi:10.1007/ s10236-012-0575-Z
Vousdoukas MI, Ferreira Ó, Almeida LP, Pacheco A (2012) Toward reliable storm-hazard forecasts: XBeach calibration and its potential application in an operational early-warning system. Ocean Dyn 62: 1001-1015. doi:10.1007/s10236-012-0544-6

Wang X, Wang Q, Sidorenko D, Danilov S, Schröter J, Jung T (2012) Long-term ocean simulations in FESOM: evaluation and application in studying the impact of Greenland Ice Sheet melting. Ocean Dyn 62:1471-1486. doi:10.1007/s10236-012-0572-2 